J.B.METZLER 
Herausgegeben

von Burkhardt Lindner

unter Mitarbeit von

Thomas Küpper und

Benjamin-

Handbuch

Timo Skrandies

Leben - Werk - Wirkung

Verlag J. B. Metzler

Stuttgart - Weimar 
Das Handbuch wurde durch Personal- und Sachmittel der Deutschen Forschungsgemeinschaft gefördert.

\section{Der Herausgeber:}

Burkhardt Lindner ist Professor für Geschichte und Ästhetik der Medien sowie für Neuere deutsche Literaturwissenschaft an der Universität Frankfurt am Main.

Bibliografische Information Der Deutschen Bibliothek Die Deutsche Bibliothek verzeichnet diese Publikation in der Deutschen Nationalbibliografie; detaillierte bibliografische Daten sind im Internet über $<$ http://dnb.ddb.de> abrufbar.

(O) 2006 Springer-Verlag GmbH Deutschland

Ursprünglich erschienen bei J. B. Metzlersche Verlagsbuchhandlung und Carl Ernst Poeschel Verlag GmbH in Stuttgart 2006 www.metzlerverlag.de info@metzlerverlag.de

ISBN 978-3-476-01985-1 ISBN 978-3-476-98691-7 (eBook)

DOI 10.1007/978-3-476-98691-7

Dieses Werk einschließlich aller seiner Teile ist urheberrechtlich geschützt. Jede Verwertung außerhalb der engen Grenzen des Urheberrechtsgesetzes ist ohne Zustimmung des Verlages unzulässig und strafbar. Das gilt insbesondere für Vervielfältigungen, Übersetzungen, Mikroverfilmungen und die Einspeicherung und Verarbeitung in elektronischen Systemen. 


\section{Inhaltsverzeichnis}

Vorbemerkung. $\ldots \ldots \ldots \ldots \ldots \ldots \ldots$ VIII

Benjamin lesen... Uber die Konzeption des Handbuchs ................... IX Von Burkhardt Lindner

Hinweise zur Benutzung............... IX

Die Werkanalysen .................... X

Im Dickicht der Texte ................. XI

Der Schriftsteller mit zwei Händen .......... XII

\section{Leben, Werk, Wirkung}

1. Zeit und Person.

Von Nadine Werner

Weltkrieg und Revolution: Auf der Suche nach

einem neuen System der Metaphysik.......... 3
Weimarer Republik: Autorschaft des

Intellektuellen im publizistischen Feld ....... 5

Exil: Erwachen aus der Urgeschichte des 19.

Jahrhunderts, der kommende Krieg......... 6

\section{Quellen und Hilfsmittel}

der Benjamin-Forschung.

Die Edition des Werks und der Briefe

(Nadine Werner) .................. 9

Bibliographien

(Sarah Steffen und Nadine Werner) ......... 10

Einführungen und Materialien

(Katharina Weber) ................... 12

Das Walter Benjamin Archiv

(Ursula Marx) .................... 15

3. Rezeptionsgeschichte

Von Thomas Küpper und Timo Skrandies

Die Konstruktion des Autors aus dem

Nachlaß......................... 17

Der Beginn der Benjamin-Rezeption,

Nachkriegszeit, 68er Kontext ............. 22

Perspektiven der theoretischen Aneignung . . . . 29

Marxismus ....................... 32

Judentum und Messianismus ............. 35

Dekonstruktion ................... 38
Literaturwissenschaft $\ldots \ldots \ldots \ldots \ldots \ldots \ldots, 42$

Genderforschung $\ldots \ldots \ldots \ldots \ldots \ldots \ldots \ldots, \ldots \ldots$

Medienwissenschaft ................ 46

Kulturwissenschaft................... 49

Benjamin als Figur des kulturellen

Gedächtnisses..................... 53

\section{Analysen}

1. Intellektuelle Freundschaft . . . . . . . . . . 59

Gershom Scholem .................... 59

Von Stéphane Mosès

Bertolt Brecht..................... 77

Von Nikolaus Müller-Schöll

Das Institut für Sozialforschung / Gretel Adorno,

Adorno und Horkheimer ............... 92

Von Christoph Gödde und Henri Lonitz

\section{Messianismus, Ästhetik, Politik}

Schriften zur Jugend ................. 107

Von Thomas Regehly

"Das Leben der Studenten « / "Dialog über die

Religiosität der Gegenwart « / "Metaphysik der

Jugend «

"Das Glück des antiken Menschen «.......... 118

Von Ansgar Hillach

Fragmente zur Ästhetik Phantasie und Farbe .. 124

Von Heinz Brüggemann

"Über das Programm der kommenden

Philosophie

Von Peter Fenves

"Der Begriff der Kunstkritik in der deutschen

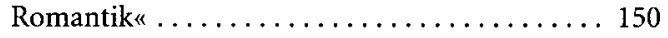

Von Justus Fetscher

"Kapitalismus als Religion «. . .

Von Uwe Steiner

Das Theologisch-politische Fragment .

Von Werner Hamacher

"Zur Kritik der Gewalt« 
"Ursprung des deutschen Trauerspiels«

Von Bettine Menke

"Das Kunstwerk im Zeitalter seiner technischen

Reproduzierbarkeit «................. 229

Von Burkhardt Lindner

Die Passagenarbeit................... 251

Von Irving Wohlfarth

Unterwegs in den Passagen-Konvoluten . . . . . 274

Von Timo Skrandies

»Über den Begriff der Geschichte«. . .

Von Jeanne Marie Gagnebin

\section{Literaturkritik, Avantgarde, Medien,}

Publizistik

"Ankündigung der Zeitschrift: Angelus Novus«.

»Zuschrift an Florens Christian Rang" ...... 301

Von Uwe Steiner

Literaturkritik

Von Michael Opitz

Zur französischen Literatur und Kultur.

Von Laure Bernardi

Zur russischen Literatur und Kultur /

»Moskauer Tagebuch".............. 343

Von Sergej A. Romaschko

"Einbahnstraße $«$. . . . . . . . . . . . . . . . . . 359

Von Gérard Raulet

Zum Kinde.

Von Giulio Schiavoni

„Programm eines proletarischen Kinder-

theaters / 》Eine kommunistische Pädagogik /

Kinderbücher

»Der Sürrealismus. Die letzte Momentaufnahme

der europäischen Intelligenz" .......... 386

Von Karlheinz Barck

"Kleine Geschichte der Photographie«

Von Eckhardt Köhn

Die Rundfunkarbeiten. ................ 406

Von Sabine Schiller-Lerg

Positionierung des Linksintellektuellen im Exil

Von Chryssoula Kambas

"Der Autor als Produzent « / Zum gegenwärtigen gesellschaftlichen Standort des französischen Schriftstellers« / "Pariser Brief» (I/II)

Anthologien des Bürgertums

Von Momme Brodersen

"Vom Weltbürger zum Großbürger« / „Deutsche

Menschen " $/$ "Allemands de

quatre-vingt-neuf« / »Carl Gustav Jochmann«
Zu Traditionskrise, Technik, Medien

Von Burkhardt Lindner

"Ich packe meine Bibliothek aus « / "Der destruk-

tive Charakter " / "Erfahrungsarmut « /

"Eduard Fuchs, der Sammler und der Historiker « /

"Lichtenberg. Ein Querschnitt « / Sur Scheerbart /

"Vereidigter Bücherrevisor"

4. Dichtungsanalyse und Autorbild

"Zwei Gedichte von Friedrich Hölderlin"...

Von Patrick Primavesi

"Goethes Wahlverwandtschaften «. Goethe im

Gesamtwerk ................... 472

Von Burkhardt Lindner

Zu Johann Peter Hebel . . . . . . . . . . . . . . 493

Von Erdmut Wizisla

Zu Gottfried Keller ................. 502

Von Erdmut Wizisla

»Zum Bilde Prousts« .................... 507

Von Ursula Link-Heer

$»$ Karl Kraus $« \ldots \ldots \ldots \ldots \ldots \ldots \ldots \ldots \ldots 522$

Von Alexander Honold

"Johann Jakob Bachofen" ................ 539

Von Sigrid Weigel

Zu Franz Kafka.................... 543

Von Sigrid Weigel

»Der Erzähler. Betrachtungen zum Werk Nikolai

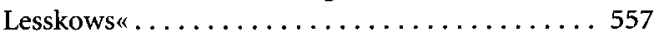

Von Detlev Schöttker

Das Baudelaire-Buch.................. 567

Von Christine Schmider und Michael Werner

"Das Paris des Second Empire bei Baudelaire" /

„Uber einige Motive bei Baudelaire / "Zentral-

park / "Notes sur les Tableaux parisiens

de Baudelaire"

5. Sprachphilosophie; literarisches und

autobiographisches Schreiben ........... . 585

Die Sonette an Heinle . . . . . . . . . . . . . . 585

Von Reinhold Görling

»Über Sprache überhaupt und über die Sprache

des Menschen".................. 592

Von Uwe Steiner

Der Brief an Buber vom 17.7.1916......... 603

Von Samuel Weber

"Die Aufgabe des Übersetzers". . . . . . . . . . . 609

Von Alfred Hirsch

Städtebilder - Reisebilder - Denkbilder

Von Roger W. Müller Farguell 
"Neapel / / Weimar « / "Marseille / / Essen $*$ /

"Nordische See« / »Kurze Schatten« (I/II) /

"In der Sonne / »Ibizenkische Folge" / "Denkbilder « / "Kleine Kunst-Stücke»

Zur späteren Sprachphilosophie. 643

Von Anja Lemke

"Lehre vom Ähnlichen / /Über das mimetische Vermögen « / »Probleme der Sprachsoziologie. Ein

Sammelreferat $\star$

"Berliner Kindheit um neunzehnhundert« ...

653

Von Anja Lemke

Aufzeichnungen

Von Manfred Schneider

Tagebücher / "Agesilaus Santander / Träume /

Drogenprotokolle / "Verzeichnis der gelesenen Schriften"

Briefe und Briefwechsel. 680

Von Gert Mattenklott

\section{Anhang}

Abbildungen....................... 689

Bildquellen....................... 696

Werkregister....................... 697

Namensregister .................... 702

Sachregister ...................... 716

Die Autorinnen und Autoren ............. 719 


\section{Vorbemerkung}

Von allen deutschen Intellektuellen der Weimarer Republik und ihres vom Hitlerreich aufgezwungenen Exils hat sich Walter Benjamin, so darf man ohne Übertreibung sagen, als der philosophisch Gewichtigste und der wirkungsgeschichtlich Lebendigste erwiesen. Die Vielfältigkeit seiner Impulse, das kontroverse Potential seiner Texte und die Radikalität seines Denkens wirken weiter fort. Davon zeugt eine breite internationale Diskussion. Seine Schriften werden in verschiedensten Disziplinen rezipiert. Die Ausstrahlung über den akademischen Bereich hinaus in die Bereiche der Gegenwartsliteratur, der Künste, der Medien und der Publizistik ist offenkundig.

Diese außerordentliche Wirkung hat sowohl mit seiner besonderen Denk- und Schreibweise zu tun als auch mit der Gestalt, in der sein Werk heute zugänglich ist. Denn mit dem Abschluß der Ausgabe der Gesammelten Schriften und mit der sechsbändigen BriefEdition (und Editionen des Briefwechsels) ist eine Textbasis erreicht, die in der ersten Hochphase der Benjaminrezeption im 68er Kontext noch völlig undenkbar erschien. Damit ist die Beschäftigung mit Benjamins Werk vor neue Aufgaben gestellt, die gegenüber der bisherigen Rezeption Vertiefungen ermöglichen und Korrekturen verlangen.

Beide Aspekte - die weitverzweigte Benjamindiskussion und die nunmehr gegebene Textbasis - sind Anlaß, dieses Handbuch vorzulegen und damit einen Zugang zu Benjamins Werk zu eröffnen, der über die begrenzten Ansprüche vorhandener Einführungen und Sammelbände grundsätzlich hinausgeht.

Das Handbuch versammelt renommierte Benjaminexpertinnen und -experten verschiedener Richtungen, verschiedener Nationalität und verschiedenen Alters in der Absicht, ausgehend vom Stand der Forschung das Spektrum der Schriften Benjamins in Einzelanalysen neu zu erschließen. Das Ganze war ein Experiment, das von Anfang an auf große Bereitschaft, sich zu beteiligen, stieß, aber auch viel Geduld bis zur endgültigen Realisierung abverlangte. Damit wird nunmehr eine umfassende Darstellung geboten, die für alle, die sich für Benjamin interessieren oder sich weiter in sein Werk einarbeiten wollen, grundlegend ist.

Das Handbuch, wie es hier vorliegt, will kein bestimmtes Bild von Benjamin durchsetzen; die Beiträge spiegeln ein Ensemble durchaus widerstreitender Lektüre-Interessen. Und es sind keineswegs nur rüberzeugte Benjaminianer, die hier zu Worte kommen. Benjamin taugt nicht zur Leitfigur. Einer Tendenz aber wird allerdings entschieden entgegengetreten: der Auffassung, daß Benjamins Schreiben im wesentlichen essayistisch, metaphorisch, literarisch ausgerichtet sei und somit ein Arsenal aparter Formulierungen biete, aus dem jedermann sich unbekümmert bedienen könne.

Dem wurde früh schon widersprochen. Es war Adornos Verdienst, die posthume Überlieferung der Schriften und des Nachlasses im Namen der Philosophie auf den Weg gebracht zu haben - einer Philosophie freilich, die sich mit den akademisch vorsortierten Grenzen nicht begnügen will.

Danken möchte ich den vielen Autoren für die intensive Kooperation, für wertvolle Gespräche und nicht zuletzt für die Beiträge selbst. Weiter gilt der Dank den Lektoren des Metzler-Verlags, Uwe Schweikert und Ute Hechtfischer, die durch Lektüre der Manuskripte und durch hilfreiche Beratung das Zustandekommen des Handbuchs sehr befördert haben. Mein besonderer Dank gilt den beiden Mitarbeitern Thomas Küpper und Timo Skrandies, die das Projekt von Anfang an mittrugen, sowie den studentischen Redaktionsmitgliedern Ute Bansemir, Inga Betten, Sarah Steffen, Katharina Weber und Nadine Werner für ihre Beiträge und ihr großes Engagement in allen Bereichen der Manuskriptherstellung; ebenso danke ich Ursula Marx vom Benjaminarchiv für ihren Beitrag und ihre Unterstützung. Zu danken habe ich schließlich der Deutschen Forschungsgemeinschaft für die großzügige Bewilligung der Förderungsmittel.

Frankfurt am Main, März 2006 Der Herausgeber 


\section{Benjamin lesen... Über die Konzeption des Handbuchs}

von Burkhardt Lindner

Ein Benjamin-Handbuch? Die Einwände ließen sich rasch aufzählen, warum gerade Benjamins Denken und Schreiben sich einer handbuchartigen ErschlieBung gänzlich entziehe. Das mag tatsächlich so sein. Aber alle, mit denen ich über diesen Plan sprach, haben spontan bestätigt, wie dringlich und wichtig ein solches Unternehmen sei. Die Konzeption, die dafür gefunden wurde, und damit auch der Gebrauchswert, den das Handbuch bieten kann, sollen im folgenden erläutert werden. Zugleich soll für den Handbuchbenutzer erläutert werden, was die Eigenart der Benjaminschen Texte ausmacht, die sehr viel darüber lehren, was lesen und schreiben, denken und wahrnehmen heißt.

\section{Hinweise zur Benutzung}

Das Handbuch ist in zwei unterschiedlich umfangreiche Teile gegliedert, die jeweils spezifische Aufgaben und auch Darstellungsweisen haben.

Der erste Teil hat den Charakter einer Einführung in Leben, Werk und Wirkung. Er wurde von der Handbuchredaktion verfaßt. Der biographische Abriß ermöglicht eine Orientierung über die wichtigsten Lebensdaten und verzeichnet chronologisch die wichtigsten Schriften. Wer mit Benjamins Werkbiographie nicht vertraut ist, kann hier nachschlagen.

Das anschließende Kapitel stellt zusammen, was inzwischen das Fundament der Benjaminforschung bildet: Bibliographien, Biographien, Einführungen, Editionen sowie das Archiv des Nachlasses. Es eröffnet damit dem Leser, der sich in das Werk und die Forschung weiter einarbeiten will, unverzichtbare Quellen und Hilfsmittel.

Daß Benjamins Werk ganz wesentlich das Resultat einer posthumen Rezeption darstellt, wird in dem umfangreichen Kapitel zur Wirkungsgeschichte behandelt. Den Ausgangspunkt bilden die Darstellung des Editionsbeginns unter den schwierigen Nachkriegsbedingungen und der Explosion der Benjaminrezeption im 68er Kontext. Daß die jüngere Rezeption dann nach 'fachwissenschaftlichen ‘ Diskursen geordnet wird, entspringt dem pragmatischen Zweck, dem Benutzer zugänglich zu machen, auf welch verschiedenen Fel- dern Benjamins Schriften in Anspruch genommen werden. Des weiteren wird auf die außerakademische Rezeption eingegangen. Gerade für diesen Autor ist kennzeichnend, daß sein Werk nicht allein im engeren Bereich einer 'Benjamin-Forschung ' wirksam wurde, sondern sich in davon abgelösten Fernwirkungen, Inanspruchnahmen, Anverwandlungen bewegte.

Die ausführlichen Literaturverzeichnisse des ersten Teils bilden zusammengenommen eine einführende Benjaminbibliographie, die dem Studierenden eine rasche Orientierung ermöglicht.

Der zweite Teil - »Analysen « - enthält die Artikel zu den wichtigsten Einzelschriften sowie Textgruppen. Wie dieser Hauptteil des Handbuchs angelegt ist, wird in den folgenden Abschnitten näher erläutert.

Schließlich kann der Benutzer über drei Registerein Namensregister, ein Sachregister und ein Werkregister - am Schluß des Handbuchs gezielt nach bestimmten Werken Benjamins, nach Personen und nach allgemeinen Sachstichworten suchen und damit Ausführungen in mehreren Artikeln vergleichend heranziehen.

Zur Zitierweise: Die Zitatnachweise aus Benjamins Werken erfolgen durchgängig nach Zitat direkt im Text. Grundlage ist die Edition der Gesammelten Schriften (hg. von Rolf Tiedemann und Hermann Schweppenhäuser). Der Nachweis erfolgt mit Bandangabe (ohne Nennung der Teilbände) als römische Ziffer und Seitenzahl.

Die Überprüfung der Benjamin-Zitate erfolgte nach der Taschenbuch-Edition der Gesammelten Schriften von 1991, da nach Auskunft der Herausgeber (vgl. dort die Editorische Notiz VII, 885 vom Januar 1991) nur sie den neuesten Stand der nachträglichen Korrekturen von Druck- und Lesefehlern bietet.

Die Zitate aus den Briefen von Benjamin werden mit Bezug auf die sechsbändige Ausgabe der Briefe (hg. von Christoph Gödde und Henri Lonitz) nachgewiesen, jeweils mit Bandangabe in arabischer Ziffer und Seitenzahl. Alle Briefe von Benjamin werden einheitlich nach dieser Ausgabe zitiert, unabhängig davon, $\mathrm{daB}$ viele Briefe bereits auszugsweise in den Anmerkungsapparaten der Gesammelten Schriften abgedruckt waren oder inzwischen auch in Editionen einzelner Briefwechsel anders zugänglich sind. 
Einzelne Texte Benjamins, die nicht in den Gesammelten Schriften abgedruckt sind, wie auch alle übrige Literaturverweise werden durch Kurzangabe (Autor, Jahr, Seitenzahl) im Artikel sowie vollständigen Titel im Literaturverzeichnis der jeweiligen Beiträge nachgewiesen.

Alle Titel, die auf Benjamin selbst zurückgehen, werden im Text durch Kapitälchen hervorgehoben. Buchtitel anderer Autoren erscheinen kursiviert; die Titel von Aufsätzen sind durch Anführungszeichen gekennzeichnet. Zitate in Zitaten werden durch einfache Anführungszeichen markiert. Weitere Kursivierungen im Text entsprechen Hervorhebungen seitens der Autoren.

\section{Die Werkanalysen}

Von Anfang an sah die Konzeption des Handbuchs vor, den Einzelschriften Benjamins einen möglichst großen Platz einzuräumen und damit alle wichtigen Texte oder Textgruppen für sich zu erschließen. Die Ausrichtung an Einzelschriften hat den Sinn, einer vereinheitlichenden Nacherzählung von Werkphasen oder der vorschnellen Herstellung eines Gesamtbildes entgegenzuwirken. Bewußt wurde deshalb auch darauf verzichtet, das Handbuch an Überblicksartikeln, die die >übergreifenden Aspekter des Werks resümieren, auszurichten. Derartige Zusammenfassungen führen letztlich von dem ab, worauf es dem Handbuch vor allem ankommt: von der Lektüre der Texte und der produktiven Auseinandersetzung mit ihnen, die in vieler Hinsicht überhaupt erst begonnen hat. Die Ausrichtung der Handbuchartikel auf Einzelschriften ermöglicht darüber hinaus, die Singularität der Textformen, den Spielraum der Schreibweisen und die Radikalität der Problemstellungen Benjamins konkret nachzuvollziehen.

Deshalb wurde auch den Autoren kein starres Abfassungsschema oktroyiert und ihnen für die eigene Werkanalyse mehr Raum gegeben, als es ein bloß lexikalisches Nachschlagewerk zuließe. Zum Nachschlagen ist das Handbuch allerdings durchaus geeignet. Der Benutzer hat in jedem Fall die Möglichkeit, sich über die Entstehungsgeschichte, die Thematik und die Rezeption der Texte zu informieren; aber er wird bei genauerer Lektüre feststellen können, daß hier in beträchtlichem Umfang anspruchsvolle Neuinterpretationen vorliegen, die die weitere Diskussion herausfordern.

Die Auswahl der Texte ist breit angelegt. Vor allem wird dabei kein Unterschied zwischen zu Lebzeiten publizierten und aus dem Nachlaß publizierten Texten oder zwischen Texten, die definitiv abgeschlossen wurden, und Texten, die unabgeschlossen blieben, gemacht. Derartige editionsphilologische Kriterien haben sich rezeptionsgeschichtlich als völlig trügerisch herausgestellt. Bestimmte Texte Benjamins haben groBes Interesse ausgelöst unabhängig davon, $o b$ sie früh oder spät entstanden, ob sie umfangreich oder kurz, ob sie zu Lebzeiten publiziert oder erst aus dem Nachlaß ediert wurden. Die sprachphilosophischen Texte oder das Tagebuch der Moskaureise oder die späten Geschichts-Thesen waren nicht zur Publikation bestimmt. Aber Benjamin hat die Texte bewahrt und damit zur Überlieferung vorgesehen. In diesem Sinne präsentiert das Handbuch, soweit es im vorgegebenen Rahmen möglich war, alle wichtigen Einzeltexte bzw. Textgruppen.

Aus analogen Gründen wurde auch eine rein zeitliche Anordnung der Artikel verworfen. Ebenso wie eine Gliederung nach Haupt- und Nebenschriften suggeriert auch eine chronologische Gliederung unvermeidlich die Vorstellung von einer fortschreitenden Werkentwicklung von den Anfängen bis zur Reife, eine Annahme, die gerade im Falle Benjamins völlig irreführend wäre.

Statt dessen werden die Schriften in fünf Abteilungen gruppiert, die nach Schwerpunkten und Schreibimpulsen, die Benjamins Arbeit lebenslang bestimmt haben und die jeweils das Gesamtwerk umspannen, gegliedert sind. Alle fünf Abteilungen sind gleich bedeutsam und gewichtig; die Frage, ob ein einzelner Artikel nicht reigentlich $\mathrm{c}$ in eine andere Abteilung gehörte, ist insofern als sekundär anzusehen.

Der erste Abschnitt "Intellektuelle Freundschaft" rückt drei biographische Konstellationen in den Blick, die Benjamin intellektuell wie persönlich ganz wesentlich geprägt haben. Wollte man diesem Stichwort in seinem ganzen Umfang gerecht werden, müßten noch weitere Personen, zu denen Benjamin intensive Beziehungen unterhielt, insbesondere auch Frauen, genannt werden. Hier sei ausdrücklich auf die Edition der Briefe verwiesen. Benjamin war ein großer Briefschreiber, dem ganz verschiedene Tonfälle und Gesten zur Verfügung standen. Und es ist kein Zufall, daß sich auch die Artikel dieser Abteilung ganz wesentlich auf Briefe und die Aufzeichnungen von Gesprächen stützen.

Unter dem Stichwort "Messianismus, Ästhetik, Politik« sind Artikel über philosophische Texte Benjamins zusammengestellt, die keineswegs unter einem einheitlichen Thema zusammenzufassen wären, sondern auf verschiedene Weise das im Titel angegebene Spannungsfeld austragen. Damit wird deutlich, daß es unproduktiv geworden ist, Benjamins Texte in der 
Konkurrenz von Marxismus und Theologie sortieren zu wollen, ebensowenig wie nach der Unterscheidung zwischen ästhetischen und politischen Untersuchungen.

Eine eigene Abteilung ist dem Literaturkritiker und Publizisten Benjamin gewidmet ("Literaturkritik, Avantgarde, Medien, Publizistik «). In diesem Bereich hat Benjamin eigene politische Schreibweisen entwikkelt, die eine erstaunliche Vielfalt zeigen und zugleich die unterschiedlichen medialen Publikationsbedingungen reflektieren. Anzumerken bleibt, daß die große Menge der Rezensionen, Feuilletonbeiträge, Polemiken, satirischen Glossen, Interviews und Rundfunkarbeiten im Rahmen des Handbuchs nicht im vollen Umfang und in wünschenswerter Detailliertheit behandelt werden konnte. Der Leser wird aber genügend Anstöße finden, um in Benjamins Texten auf eigene Entdeckungsreise gehen zu können.

Die vierte Abteilung »Dichtungsanalyse und Autorbild " präsentiert Artikel zu den sliterarischen Essays Benjamins. Auch sie sind, ihrer Textform wie ihrer Thematik nach, nicht einheitlich; was sie aber verbindet, ist die Intensität, mit der sie jeweils auf einen einzelnen Autor bzw. auf ein einzelnes Werk eines Autors ausgerichtet sind. Benjamin hat eine besondere Meisterschaft entwickelt, strenge Dichtungsanalyse mit porträthaften biographischen Zügen zu verknüpfen, ohne damit einer psychologistischen Werkhermeneutik zu verfallen.

Die Artikel der letzten Abteilung »Sprachphilosophie; literarisches und autobiographisches Schreiben" rücken im engeren Sinne autobiographische Texte ebenso wie Aufzeichnungen und Briefe sowie die Sonette mit den grundlegenden sprachphilosophischen Texten zusammen, die zum wesentlichen unpubliziert blieben. Zwar muß man sagen, daß es keinen Text Benjamins gibt, der ohne Reflexion auf die Medialität von Sprache, Schreiben und Schrift wäre, aber es erschien gerade für die Zwecke des Handbuchs wichtig, diesen Komplex durch eine eigene Abteilung hervorzuheben. (Ein vorgesehener Text zu Benjamin als Ubersetzer ist nicht zustandegekommen.)

Das Handbuch bietet fünf querschnittartige Durchgänge durch das Werk, die damit thematische Konstellationen ergeben. Vom Benutzer/Leser des Handbuchs wird erhofft, daß er nicht nur einzelne Artikel nachschlägt, sondern Lust bekommt, selbst Beziehungen herzustellen, die über die Anordnung der Autorenartikel hinaus- und in das Gesamt der hinterlassenen Schriften hineinführen.

Das Erstaunliche der Benjaminschen Produktion besteht ja darin, daß alles Geschriebene eine intellektuelle und stilistische Unverkennbarkeit hat. Alle Texte führen einen geheimen Kompaß mit sich, sie verweisen auf das verborgene Gravitationszentrum seines Denkens und Schreibens. Alle Texte unterhalten geheime Verbindungen, enthalten teilweise Selbstzitate, nehmen frühere Problemstellungen wieder auf, wandeln sie $a b$, konstellieren sie neu. Jeder, der sich intensiv in Benjamins Schriften versenkt, wird diese Erfahrung machen. Es ist Absicht des Handbuchs, zu dieser Erfahrung einzuladen.

\section{Im Dickicht der Texte}

Die Konzeption des Handbuchs sucht der besonderen Gestalt des Werks von Benjamin Rechnung zu tragen, die erst eigentlich posthum - durch Erschließung des verstreut Publizierten und Transkription des umfangreichen Nachlasses - erkennbar wurde. Über die abenteuerliche Geschichte der Überlieferung und der Nachlaßfunde, die noch in die lange Editionsgeschichte der Gesammelten Schriften hineingewirkt hat, muß hier nichts gesagt werden (s. dazu den Teil "Rezeptionsgeschichte (). Dank Rolf Tiedemann, der die immense Entzifferungs- und editorische Sicherungsarbeit der Gesammelten Schriften im wesentlichen trug, können die Benjaminleser heute auf eine so gut wie vollständige, philologisch genaue Textgrundlage zurückgreifen, wie es sie sonst für keinen philosophischen Autor seiner Generation gibt. In ihr ist der gesamte Nachlaß erfaßt und auch das, was nicht oder nur auszugsweise publiziert wurde, verzeichnet.

Für das Handbuch bedeutete dies, daß überall die Textgrundlage der Gesammelten Schriften zugrundegelegt wird. Nur in einzelnen rezeptionsgeschichtlich wichtigen Fällen wird auf frühere Ausgaben gesondert verwiesen. Hingegen konnte sich die Anordnung der Werkanalysen im Handbuch nicht an den Gliederungsprinzipien der Gesammelten Schriften orientieren. Es wäre gewiß einfacher gewesen, die Anordnung parallel zu der nun einmal vorgegebenen (und dem versierten Benjaminleser vertrauten) Gliederung folgen zu lassen. Aber, auch ohne hier in eine Diskussion der Gliederungsprinzpien eintreten zu wollen, kann festgestellt werden, daß sie eine höchst problematische Aufteilung in Werkformen und Textsorten unternimmt, die zudem durch die späteren Nachtragsbände (VII/1 und 2) und den umfangreichen Nachlaßband (VI) vollends unübersichtlich geworden ist.

Benjamins Werk besteht in seinem Hauptteil aus Einzeltexten; allein die als abgeschlossen geltenden Arbeiten umfassen über 500 Titel. Hingegen konnte Benjamin zu Lebzeiten nur vier Bücher publizieren. Und doch sollte man sich hüten, das durch Zeitum- 
stände Verwehrte - Benjamin hat selbst einmal von der "Katastrophen- und Trümmerstätte" seines Schreibens gesprochen - zum Anlaß zu nehmen, das Überlieferte im Bild der Ruine oder des Fragments zu stilisieren. Was auf den ersten Blick als Disparatheit und Verstreutheit erscheint, hat sich längst als ein Kraftfeld erwiesen, demgegenüber jede Vorstellung, Benjamin habe die reigentlichen Werke r nicht schreiben können, völlig verblaßt. Bei ihm wie sonst wohl bei niemandem anders ist die Qualifizierung von Hauptwerken und Nebenarbeiten völlig hinfällig, ohne daß etwa die textanalytische Unterscheidung zwischen einem Aphorismus, einer Rezension und einer umfangreichen $\mathrm{Ab}$ handlung deshalb unwesentlich wäre.

Dem Leser wird damit abverlangt, sich diesem Dikkicht der Texte auszusetzen. Das Handbuch will nicht nur allgemein zur Lektüre der Benjaminschen Texte anregen, sondern ausdrücklich zur extensiven Benutzung der Edition der Gesammelten Schriften auffordern. Es genügt hierzu allerdings nicht, auf das außerordentlich akribische "Gesamtinhaltsverzeichnis" (VII, 899 - 1019), das in fünf gesonderten Registern alle abgedruckten Texte der Gesammelten Schriften erschließt, zu verweisen. Wer sich nicht auskennt, wird hier rasch ratlos werden.

Um dem Leser auf die Sprünge zu helfen, wird statt dessen im Handbuch allen Werkanalyse-Artikeln jeweils ein Werkverzeichnis nachgestellt. Die Werkverzeichnisse informieren den Benutzer über die für den Beitrag einschlägigen Texte Benjamins. Insbesondere werden hier auch die in den Apparaten und im Nachtragsband versteckten Nachlaßteile - Paralipomena, Arbeits-Schemata, ausformulierte Notizen, Literaturlisten - aufgelistet. Da Benjamin das Material seiner Manuskripte wohl so gut wie nie vernichtet hat, bilden diese Nachlaßteile vielfach ein einzigartiges Archiv des Schreibprozesses. Darüber hinaus wird im Werkverzeichnis auf weitere Texte hingewiesen, die in engem Bezug zu dem im Artikel behandelten Haupttext stehen, aber in der Schriftenausgabe nicht an diesem Ort zu finden sind. So wird über die Ausführungen im Autorartikel hinaus ein konstruktiver Zugang zu den Gesammelten Schriften eröffnet.

\section{Der Schriftsteller mit zwei Händen}

»In jeder Epoche muß versucht werden, die Überlieferung von neuem dem Konformismus abzugewinnen, der im Begriff steht, sie zu überwältigen. « Es wäre zu billig, diesem philosophischen Imperativ aus den Thesen Úber den Begriff der Geschichte den exzeptionellen Nachruhm ihres Autors entgegenzuhalten - und täte man es, würde es nicht Benjamin sondern vielmehr die gedankenlosen Kulturagenten treffen, die das Werk konjunkturentsprechend verwerten.

Was mit der ersten Phase kontroverser Benjaminaktualisierung im 68er Kontext begann - notwendig auch mit überzogenen, gegenüber seinem historischen Kontext unkundigen Aktualisierungen -, hat sich mit neuen Problemstellungen und Akzentverschiebungen fortgesetzt. Inzwischen ist beträchtliche theoretische wie historische Forschungsarbeit geleistet worden. Und die politische Erosion der letzten 25 Jahre hat das Interesse an Benjamin nicht erlahmen lassen. Im Gegenteil könnte man sagen: seine Texte haben diese Diskursverschiebungen nicht nur unbeschädigt überstanden, sondern kehren ihre Widerständigkeit neu hervor, wie die Beiträge des Handbuchs erweisen und mit neuen Impulsen versehen. Den Funktionären des täglichen Fortschritts in der Katastrophe hat Benjamin allemal in die Suppe gespuckt.

Benjamin verkörpert auf herausragende Weise die Figur des europäischen, deutschjüdischen Intellektuellen, und damit eine brüchige Tradition, die heute wieder in Vergessenheit zu geraten droht. Wenn seine Schriften diesem Vergessen Widerstand bieten, so beruht dies nicht allein auf bestimmten Theoremen, Motiven, Themen und Überzeugungen, sondern auf der Eigenart seines Denkens und Schreibens, dessen Originalität sich gerade darin erweist, daß er zwar gewiß Bewunderer, aber weder gläubige Schüler noch erfolgreiche Nachahmer gefunden hat. Jeder Leser Benjamins, der über das Gelesene schreiben will, wird die Erfahrung machen, daß die Texte sich der Inhaltszusammenfassung entziehen. Ohne die Prägnanz der Formulierung, die einem außerordentlichen Bewußtsein der deutschen Sprache entspringt, verflüchtigt sich ihr Wahrheitsgehalt, weshalb in der Öde mancher Sekundärdarstellung ein Benjaminzitat wie ein strafender Lichtblick wirkt.

Diese sprachliche Souveränität schloß Verknüpfungen ein, die auch den Freunden bedenklich oder gar zerstörerisch erschienen. Aber es ist nicht Mystifikation oder rhetorische Maskierung, sondern nüchterne Erkenntnis der eigenen Schreibkompetenz, wenn Benjamin gegen Scholems beunruhigte Warnungen darauf beharrt, "die rote Fahne zum Fenster herauszuhängen " und vom Parteistandpunkt aus "'gegenrevolutionäre، Schriften " zu verfassen, ließe sich durchaus kombinieren (Brief an Scholem vom 17.4. 1931: 4, 25).

Den Anspruch - und das Risiko - eines Denkens, das sich bewußt in Extremen bewegt, hat er in einem denkwürdigen Brief vom Juni 1934 an Gretel KarplusAdorno (»Felizitas «), die sich wegen Brechts verhäng- 
nisvollem Einfluß auf Benjamin besorgt zeigte, aufs genaueste beschrieben. «In der Ökonomie meines Daseins spielen in der Tat einige wenige gezählte Beziehungen eine Rolle, die es mir ermöglichen, einen, dem Pol meines ursprünglichen Seins entgegengesetz $[t]$ en zu behaupten. Diese Beziehungen haben immer den mehr oder weniger heftigen Protest der mir nächststehenden herausgefordert, so die zu $\mathrm{B}$ [recht] augenblicklich - und ungleich weniger vorsichtig gefaßt den Gerhard Scholems. In solchem Falle kann ich wenig mehr tun, als das Vertrauen meiner Freunde dafür erbitten, daß diese Bindungen, deren Gefahren auf der Hand liegen, ihre Fruchtbarkeit zu erkennen geben werden. Gerade Dir ist es ja keineswegs undeutlich, daß mein Leben so gut wie mein Denken sich in extremen Positionen bewegt. Die Weite, die es dergestalt behauptet, die Freiheit, Dinge und Gedanken, die als unvereinbar gelten, neben einander zu bewegen, erhält ihr Gesicht erst durch die Gefahr. Eine Gefahr, die im allgemeinen auch meinen Freunden nur in $\mathrm{Ge}-$ stalt jener 'gefährlichen $>$ Beziehungen augenfällig erscheint " $(4,440 \mathrm{f}$. $)$.

Und ein Jahr später, diesmal geht es um die Besorgnis der Adornos, das Exposé der Passagenarbeit von 1935 verleugne die metaphysisch-theologischen Impulse der ersten Entwürfe, heißt es wiederum in einem Brief an Gretel Karplus-Adorno: »Meinen Gedankenflug höher lenkend, muß ich doch bei meiner Person noch einen Augenblick verweilen. Wenn Du nämlich von meinem szweiten Entwurf schreibst )darin würde man nie die Hand WB's erkennen', so nenne ich das doch ein wenig geradezu gesagt [...]. Der WB hat - und das ist bei einem Schriftsteller nicht selbstverständlich - darin aber sieht er seine Aufgabe und sein bestes Recht - zwei Hände. Ich hatte es mir mit vierzehn Jahren eines Tages in den Kopf gesetzt, ich müsse links schreiben lernen. Und ich sehe mich heute noch Stunden und Stunden an meinem Schulpult in Haubinda sitzen und üben. Heute steht mein Pult in der Bibliothèque Nationale - den Lehrgang so zu schreiben habe ich da auf einer höhern Stufe - auf Zeit! - wieder aufgenommen. Willst Du es nicht mit mir so ansehen, liebe Felizitas? Ausführlicher will ich hierzu nicht gerade sein « (Brief vom 1.9.35: 5, 151).

Nochmals gesagt: es wäre verfehlt, derartige Selbstauskünfte als ein Versteckspiel im Raum des brieflichen Austauschs zu betrachten. Ohne Zweifel hätte Benjamin diese Formulierungen in einem für die Publikation gedachten Text nicht gebraucht. Aber ihm ist der Gedanke, daß sein Denken und Schreiben sich in heterogenen Konstellationen bewegt, ganz selbstverständlich. Benjamin hat damit nicht nur Denkverbote unterlaufen, sondern auch politische Desillusio- nierungen besser verarbeiten können als viele andere Intellektuelle seiner Generation.

In einem Korpus von Aufzeichnungen aus dem Ende der 20er Jahre, die sich in der Sammlung Scholems fanden, deren Kontext aber leider nicht genau zu ermitteln ist, notiert er: "Schriftstellern heißt Gedankenkonzerte geben." (VII, 877) Im Fortgang vergleicht er die Gedanken mit den Instrumenten eines Orchesters. "Autorenköpfe [müssen] mit Instrumenten viel besser besetzt sein, als der flüchtige Leser annimmt. Nur verhalten sie sich die meiste Zeit über schweigend um erst an der gegebenen Stelle genau, unter dem Stab des Dirigenten, einzufallen « (VII, 877). Die Kunst des philosophischen Schriftstellers besteht im präzisen Weglassen von Gedanken und Wörtern (VI, 209).

Was Benjamin (in den zitierten Briefen) als produktive Konstellation seines Daseins beschreibt, erscheint hier als innere Ökonomie der Textproduktion. In der Einbahnstrasse, in dem Stück Technische NotHILFE, findet sich eine analoge Überlegung zur Instrumentierung der Autorenköpfe. »Wer wollte die Alarmsignale zählen, mit denen das Innere des wahren Schriftstellers ausgestattet ist? Und schreiben heißt nichts anderes als sie in Funktion zu setzen « (IV, 138). In Funktion setzten bedeutete aber nichts anderes als Komposition, Kalkulation, Rhythmisierung, die vom »Objektiv der Schrift « (ebd.) diktiert wird. Denn es "gibt nichts Ärmeres als eine Wahrheit, ausgedrückt, wie sie gedacht ward « (ebd.).

Im Kontext der Passagenarbeit hatte Benjamin dazu schließlich notiert: »Die Konkretion löscht das Denken, die Abstraktion entzündet es. Jede Antithetik ist abstrakt, jede Synthesis konkret. (Die Synthesis löscht das Denken) « $(V, 1033)$. Jeder Text hat die Figur einer Stillstellung des Denkens und gibt die Löschung an den Leser weiter, der wiederum auf die "tausend Tore" (VII, 877) der Worte stößt.

Schriftstellern heißt aber nicht bloß, Gedanken zum Druck zu befördern, sondern erst einmal Schriften, Manuskripte, zu erstellen. Benjamin, der nie eine Schreibmaschine benutzte, hat der Praxis der eigenen handschriftlichen Aufzeichnung in der EINBAHNSTRASSE mehrfach eindringliche Reflexionen gewidmet (IV, 102; 106f; 112f.; 131). Daraus wird ersichtlich, warum er immer wieder mit bestimmten Manuskriptformen und Aufschreibweisen experimentiert hat. Deshalb bildet der umfangreiche Nachlaß der erhaltenen Manuskripte einen integralen Bestandteil des Werks. 